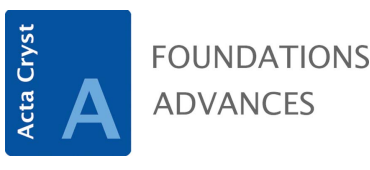

ISSN 2053-2733
Keywords: book review; basic crystallography.

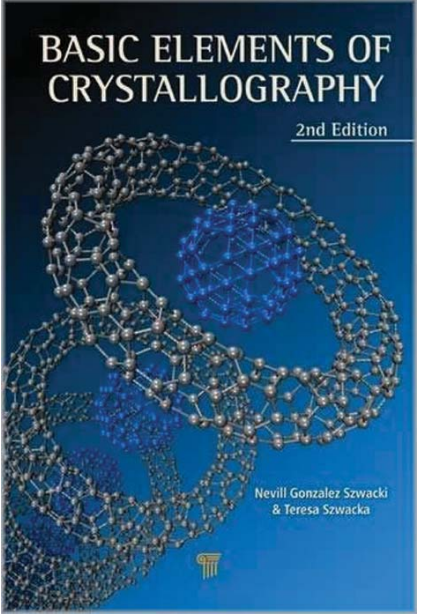

(C) 2017 International Union of Crystallography

\section{Basic Elements of Crystallography. 2nd edition. By Nevill Gonzalez Szwacki and Teresa Szwacka. Pan Stanford Publishing, 2016. Pp. $x+324$. Price USD 79.95 (paperback). ISBN 9789814613576.}

\author{
Massimo Nespolo* \\ Université de Lorraine, CRM2, UMR 7036, Vandoeuvre-les-Nancy, F-54506, France, and CNRS, CRM2, UMR 7036, \\ Vandoeuvre-les-Nancy, F-54506, France. *Correspondence e-mail: massimo.nespolo@crm2.uhp-nancy.fr
}

The first edition of this book, published in 2010, was reviewed by Nespolo (2012). I describe here only the changes with respect to the previous edition. Unfortunately, most of the defects and errors contained in the first edition are still there, and the only serious mistake that has been corrected is the wrong expression 'trigonal lattice', which is now replaced by 'rhombohedral lattice'.

The main additions and changes with respect to the first edition are the following:

(1) a new section in Chapter 1, about Wyckoff positions;

(2) four new sections in Chapter 3; one about 'symmetry axes', where the graphical symbols for direct, inverse and screw axes are listed, and three about specific structure types ('arsenic structure', 'selenium structure', 'graphite structure');

(3) a somewhat expanded section about 'interstices in close-packed structures', again in Chapter 3;

(4) the correction of an error in an equation (denominator 4 instead of 8 in Eq. 3.15, or III.12 in the previous edition);

(5) a new section in Chapter 5 ('Introduction' to the examples of reciprocal lattice);

(6) a whole new chapter (Chapter 7) on 'X-ray diffraction';

(7) the solution to the exercises, now published as Appendix; and

(8) some significant changes in the text, in particular in Chapter 1.

The concept of a conventional cell, which was undefined in the previous edition, is now defined (Chapter 1) and used with more care. Unfortunately, the definition is still vague and as a consequence its use is still partly incorrect. Let us remind the reader that a conventional cell is defined by three conditions: (1) its basis vectors define a right-handed axial setting; (2) its edges are along symmetry directions of the lattice; (3) it is the smallest cell compatible with the above conditions. In this book it is instead simply defined as the unit cell 'chosen such that the symmetry of the crystal structure is displayed best', which leads to errors and misunderstandings, as shown by the two following examples:

(i) a primitive unit cell of an oblique two-dimensional lattice is called 'conventional' ( $p$. 9) despite that fact that this lattice, having only binary rotations as non-translational symmetry operations, does not contain any symmetry directions of the lattice [condition (2)], so that a conventional unit cell is simply undefined;

(ii) the primitive rhombohedron is considered the conventional unit cell of a rhombohedral lattice (p. 74 and p. 81), which is wrong, the conventional unit cell of a rhombohedral lattice being hexagonal.

Another mistake occurs in Chapter 1, when it is stated that in the case of a centred rectangular lattice in two-dimensional space 'there is no primitive unit cell with the point symmetry of a rectangle', which is contradicted by the rhomb-shaped unit cell in Fig. 1.19 (c) whose symmetry is $2 \mathrm{~mm}$, precisely like the centred cell. The symmetry directions of the lattice are parallel to the edges of the centred cell but to the diagonals of the primitive cell, which explains why only the former is conventional. But to understand this, a precise definition of conventional cell is necessary.

The new Table 1.1 presents the symmetry elements in two-dimensional space, calling glide lines ' $g$ or $a$ ' and ' $g$ or $b$ ' (which is not consistent with the International Tables for Crystallography), and adding an inversion centre (which does not exist in every space 
with even number of dimensions). Below the table we read that the inversion centres 'coincide with twofold rotation points', which just adds confusion: operations of first and second kind simply cannot 'coincide'.

There are also mistakes in the new section on Wyckoff positions, in the definitions of general and special positions. These are defined, respectively, as having points that are located out of any or on at least one symmetry element. This is obviously contradicted by placing an atom on a g-glide line (we are still in Chapter 1, where only one- and twodimensional spaces are presented), which is on a symmetry element yet is in general position. Another example of lack of rigour comes just at the end of the chapter, when objects on which a reflection has acted are described as having changed 'orientation' instead of 'handedness'.

In the revised text of Chapter 2 we read that 'lattices that have only one sixfold rotation axis belong to the hexagonal and trigonal crystal systems'. What is meant here is that structures whose lattice is hexagonal may belong to the trigonal or hexagonal crystal system, depending on the structural symmetry; when applied only to lattices, the statement does not make sense.

The reader will struggle to follow the sentence 'The symmetry of the conventional unit cell has to be at least as high as the point symmetry of the lattice belonging to the crystal system to which belongs the crystal structure in consideration' (my emphasis). The statement that follows ('in the case of the trigonal system is used a rhombohedral or a hexagonal lattice, depending on the case') also has problems one can use either rhombohedral or hexagonal axes to describe both the rhombohedral and the hexagonal lattices $(R, P$ or $D$ unit cells), but one cannot use a lattice at will.

In the first edition, Chapter 4, about binary compounds, contained precise but not referenced data about the ionic and covalent contribution to the chemical bond: $62 \%$ covalent in $\beta$ - ZnS (removed in this new edition), $18 \%$ ionic in $\beta$-SiC (reduced to $15 \%$ in this new edition). Where these figures come from, and why they have changed, is not stated.

The brand new chapter about X-ray diffraction justifies the presence of the preceding two chapters on direct and reciprocal lattices, which were a sort of dangling bond in the previous edition. This chapter is only 19 pages long and introduces classical concepts: Laue equations, Ewald sphere (called 'construction'). Bragg's law, structure factors. The presentation is affected by the common mistake of considering Miller indices to be restricted to relatively prime indices independently from the basis vectors chosen (see a discussion in Nespolo, 2015), which leads to the selfcontradictory use of both (100) and (200) notation to indicate different planes of the same family. From a single example (bcc structure) the integral reflection conditions are introduced without further explanation; zonal and serial conditions are not mentioned.

The solution to the exercises is certainly a welcome addition, although a number of tedious calculations could have been avoided by the use of the metric tensor, which is not mentioned in this book.

In conclusion, the publication of a new edition of this book offered the chance to correct a number of mistakes and inconsistencies present in the first edition, and to include something more useful. Unfortunately, this opportunity has been almost completely missed.

\section{References}

Nespolo, M. (2012). Acta Cryst. A68, 430-432.

Nespolo, M. (2015). J. Appl. Cryst. 48, 1290-1298. 